Hollins Martin, C.J., Snowden, A., Martin, C.R. (2012). Concurrent analysis: Validation of the domains within the Birth Satisfaction Scale. Journal of Reproductive and Infant Psychology. 30(3): 247-260. http://dx.doi.org/10.1080/02646838.2012.710833

Caroline J. Hollins Martin a , Austyn Snowden b \& Colin R. Martin b

a School of Nursing, Midwifery and Social Work, College of Health and Social Care, University of Salford, Salford, Greater Manchester, UK

b School of Health Nursing and Midwifery, University of the West of Scotland, Ayr, UK

\begin{abstract}
Background and aim: Measuring women's satisfaction with their birth experience has been problematic. Recently an attempt has been made to capture birth satisfaction's generalised meaning and incorporate it into an evidenced-based tool. Standard procedures for validation have limitations. Qualitative techniques such as domain analysis offer an alternative and assist in better understanding the importance of each item. This paper examines the parsimony of the Birth Satisfaction Scale (BSS), which is a 30 item questionnaire designed to measure satisfaction with childbirth, with women's actual experience of childbirth.
\end{abstract}

Methods. Primary free text data collected from 207 women who original tested the BSS was concurrently analysed with firsthand accounts of birth satisfaction collected from 19 qualitative papers.

Results: The domain analysis confirmed three explanatory items within the BSS: 'being in control', 'things going as planned', and 'being supported'.

Conclusions: The BSS accounts for all the analysed data, suggesting it is a robust measure of satisfaction in childbirth. Strengths and limitations of the method are discussed, as are implications for practice. With further development, the instrument could be used to establish correlates with other psychometric measures, i.e., selfefficacy, anxiety, depression, locus of control and bonding; and evaluate models or care systems as a stand-alone instrument, or as a screening test prior to detailed qualitative work. 


\section{Concurrent analysis: validation of the domains within the Birth Satisfaction Scale Introduction}

The Birth Satisfaction Scale (BSS) entails 30 Likert questions (Figure 1). It was developed by Hollins Martin and Fleming (2011) to construct a meaningful picture of what constitutes like or dislike of childbearing women's experiences of labour and its outcomes. In keeping with the views of Redshaw (2008), it was considered that reports would help maternity care researchers compare experiences of consumers at both an individual level, between institutions and across cultures. The BSS may be used to: (1) identify aspects of birth dissatisfaction that may be remedied with good psychosocial care, (2) establish correlates with other psychometric measures, i.e., self-efficacy, anxiety, depression, locus of control and mother/infant attachment, (3) evaluate models or systems of care as a standalone instrument or as a screening test prior to in depth qualitative work.

Figure 1 about here

The 30 items on the BSS were constructed from a literature review of childbearing women's views of what they considered gave them birth satisfaction or dissatisfaction. Three overarching themes were identified (table 1): service provision (home assessment, birth environment, support, relationships with health care professionals); personal attributes (ability to cope during labour, feeling in control, childbirth preparation, relationship with baby); and stress experienced during labour (distress, obstetric injuries, receiving sufficient medical care, obstetric intervention, pain, long labour and baby’s health) (Hollins Martin \& Fleming, 2011) .

Table 1 about here

One of the routine methods of questionnaire validation is item analysis/factor analysis. However, there are a number of limitations to this approach in relation to questionnaire data, which may be non-normally distributed item associations that have relevance and importance to a number of domains (Kline, 2000). Further applicability to broad and diverse populations where implicit aspects of culture and society may 
profoundly impact on the measurement characteristics of the tool has been demonstrated to limit replicability of factor structures (Allan and Martin, 2009).

This paper takes an original approach to explore the detail of the BSS structure by concurrently analysing the literature on birth satisfaction simultaneously with free text data from validity testing of the scale. The purpose of this analysis was to establish the parsimony of the BSS items with women's actual experience of childbirth. The result was a qualitative domain analysis that illustrates the weight and importance of each BSS item. It also illuminated those aspects of satisfaction not necessarily covered by the scale.

\section{Aim}

The aim of this study was to establish the parsimony of the BSS with women's actual experience of childbirth.

\section{Method}

Concurrent analysis is a method of analysing narrative data for the purpose of articulating salient connections (Snowden \& Atkinson, 2012). The method uses Thagard's concept of coherence to connect representations on the basis of analogy or explanation (Thagard, 2007). The method will be expanded step by step in the analysis section. In brief, it uses any relevant first hand narrative data as primary data. This includes interview data, free text questionnaire responses or even case study and reflective literature (Snowden \& Martin, 2010b). This data is then coded in order to summarise pertinent aspects of this narrative. Often, pieces of narrative can be coded at more than one code, suggesting connections between codes, allowing deeper analysis of the experience under investigation. The product is a qualitative domain analysis that accounts for frequency, analogy and explanation of all the narrative data. This level of analysis equates with parsimony in qualitative analysis (Cutcliffe \& Harder, 2009).

\section{Primary data sample}

The initial pilot of the BSS took place in the West of Scotland in 2011. Participants were postnatal women ( $n=207)$ who gave birth at term (37-42 weeks gestation) and were willing to complete the BSS within their 
first 10 postnatal days. The study was approved by the National Health Service (NHS), National Research Ethics Service (NRES). During the validation of the BSS women were asked to provide further free text comments, and this is part of the data analysed in this study.

The primary research method used in this study was a survey using the BBS developed by Hollins Martin and Fleming (2011). A space was included after the relevant questions for the woman to write clarifications. The comments made by the women represent the overall qualitative data considered in this paper, with the quantitative analysis of the data collected by the quantitative questions to be reported elsewhere. The 30 items on the BSS were scored on a 5-point Likert scale based upon level of agreement or disagreement with each of the statements. A space was included after each question for the woman to add comments. For example:

(27) The staff communicated well with me during labour.

\begin{tabular}{|c|c|c|c|c|}
\hline $\begin{array}{l}\text { Strongly } \\
\text { Agree }\end{array}$ & Agree & $\begin{array}{l}\text { Neither Agree } \\
\text { or Disagree }\end{array}$ & Disagree & $\begin{array}{l}\text { Strongly } \\
\text { Disagree }\end{array}$ \\
\hline
\end{tabular}

Comments

\section{Firsthand data sources}

The literature provided detailed firsthand accounts of satisfaction in childbirth. The type of data required for concurrent analysis is firsthand accounts (Snowden \& Martin, 2010a). The literature can provide a rich source of such data. For the purpose of concurrent analysis first hand data published in high quality peer reviewed journals was considered conceptually equivalent to primary data such as that generated by the 207 women in the pilot study. The endpoint of this analysis is a more thorough integration of the wider literature than would usually be the case by integrating this literature at a later stage (Snowden \& Atkinson, 2012).

CINAHL, Medline, Psychinfo were searched for ‘birth satisfaction', limited to peer reviewed papers post 2004. Salient other references were chased up. One hundred and twenty five abstracts were checked for potential pertinent primary data from this search. Pertinent data included any firsthand narrative account of childbirth where satisfaction was discussed. Papers were selected for analysis on the basis of depth of description and quantity of relevant primary data they contained. At the end of this process 19 papers were 
selected. Please see Table 2 for a summary of this data. These papers were imported into NVivo9 along with the pilot free text data.

Table 2 about here

\section{Analysis}

Step 1. The coding framework

Previous concurrent analyses have developed theory directly from the data (Snowden \& Martin, 2010b; Snowden et al, 2011). However, in this case the purpose of the analysis was to test existing theory; the BSS structure. In order to achieve this, the BSS items (Figure 1) were used as a coding framework.

Two nodes were created for each BSS item, one for the BSS item and another for its opposite. The purpose of coding both positive and negative instances for each item was to generate information on both the volume of coding at any particular item and also the balance of it. For example 'I coped well during birth' is item 1 on the BSS. We constructed an opposite item: 'I didn’t cope’. All relevant narrative could therefore be coded at one point or the other. The rationale for coding in this binary manner was to generate both a frequency for all the coding at this item, and a ratio (positive: negative) indicative of the balance of this particular experience.

A further node was also created in order to capture any narrative that fell entirely outside the BSS structure. The role of this code was to identify issues that the BSS may not explicitly capture.

\section{Step 2. The coding process}

The first analytic step was to reduce the narrative data to manageable units. This is done by line by line coding, a staple of many qualitative methods (Flemming, 2010). In order to remain conceptually consistent the coding was focused on the expression of satisfaction or dissatisfaction with childbirth. This could be either process based ('They never had a break and work very hard under strenuous conditions') or meaning based ('I am very happy about my experience'), as the BSS entailed both these perspectives. This method of coding different conceptual issues together deviates from grounded theory (Charmaz, 2009) or 
phenomenology (Shattell, McAllister, Hogan, \& Thomas, 2006) for example, where the focus of the coding would be on either social process (grounded theory) or interpreted meaning (phenomenology). However, it fits with a pragmatic and emancipatory view of qualitative analysis more generally (Thorne, 2011), and is coherent with pragmatic purpose of qualitative research (Cutcliffe \& Harder, 2009).

In practice this meant the narrative was coded for every instance of issue, feeling or discussion of the birth experience in general. Table 3 shows examples from the primary data in relation to the code 'I felt out of control during my childbirth’.

Table 3 about here

Some of these statements also related to other items on BSS and were therefore also coded there. For example the first primary data example ('not what I intended' M213) was also coded at the BSS item 'my birth didn’t go as planned'. This process was undertaken for all the narrative identified in the sample.

\section{Step 3. Analysis of coding: frequency}

The end of step 2 resulted in over 2500 coding references, with many pertaining to multiple codes. Some of these codes fell outside the BSS structure, suggesting at first analysis that the BSS did not account for all experiences pertinent to birth satisfaction. This will be discussed further. In terms of frequency, the range (least to most coded BSS items) were 10 codes - 200 codes. The most coded content was at:

1. Being supported by staff (200 coding instances in total: 171 positive plus 29 negative)

2. Feeling in/out of control (191 coding instances)

3. Receiving medical attention (168 coding instances)

4. Communication (151 codes)

This shows that these are the factors most mentioned. However, in order to develop an understanding of the quality of these experiences it was also important to understand what other codes were also coded at these and other nodes. 
NVivo9 allows for cross-referencing to illuminate associations between codes. The output of this is a 'coding matrix', a spreadsheet of coding frequency at all intersections between codes. This coding matrix was exported to Microsoft excel 2007 in order to further analyse and explore these associations. As an example figure 2 shows the frequency of coding that also took place at the code 'I felt well supported by staff'.

Figure 2 about here

Figure 2 shows that women related being 'well supported' with being 'communicated with', receiving 'outstanding medical care', 'being calm’ and 'in control' and so on. In particular, 'I felt well supported by staff during my labour and birth' was highly associated with 'the staff communicated well with me during labour', in that 84 codes were cross-referenced. This procedure was repeated iteratively until all major connections had been analysed, modelled and accounted for.

Step 5. Pattern analysis: heat maps, coding distribution items coded outside BSS

The next stage was to ascertain general patterns within the overall coding. To do this, the matrix was converted to a heat map in excel. This generated a visual representation of the coding density at every intersection of every node. There is too much detail to illustrate this within a diagram for a journal, but to show the principle figure 3 highlights the intersections of a selection of 8 nodes.

Figure 3 about here

Figure 3 illustrates two important properties of the data: frequency (table of figure 3) and associate code distribution (percentage spread in the bars). The frequency table shows that being 'well supported by staff' is frequently also coded at all 4 illustrated codes. This suggests that being 'well supported by staff' is a commonly cited, potentially generalisable aspect of birth satisfaction. Support is commonly associated with satisfaction in childbirth (Hollins Martin, 2008; Redshaw, 2008). What the concurrent analysis adds is further evidence that having the same midwife throughout the process, and receiving outstanding medical care (figure 3) facilitates this. 
The associate code distribution of each bar shows the relative make up of contributing codes, and this is important as it demonstrates the comparative significance of particular associations. Because concurrent analysis is essentially qualitative in regard to its use of narrative data, the strength of association between representations is as important as the frequency in particular instances. For example, distress is highly associated with an unhealthy baby, regardless of the quality of care or support received (Beck, 2006). So, although some outcomes happen less frequently than others, their relative impact upon satisfaction may be significantly greater than other factors when they do. This is where concurrent analysis offers advantage over more quantitative methods of questionnaire validation, as the context underpinning these associations is maintained and therefore explicated as part of the analysis.

The item on the right of figure 3: 'it was a loving experience' was not a BSS item, but a direct quote from one of the women in the primary data. It wasn't immediately obvious that this could be associated with a BSS item so a new node was created called 'it was a loving experience'. In total, around 300 codes were created that appeared to sit outside the BSS structure at first sight. Examples of content coded at 'it was a loving experience’ were:

I heard just one cry, and there was a flood of tears. And I felt like I had never been happier in my entire life. I just felt more happiness and more joy and more love than I'd ever felt.p510 (Callister, 2004)

...'wonderful,' 'like a dream,' 'perfect,' (Bayes, Fenwick, \& Hauck, 2008)p57

Skin to skin straight away, bonded straight away (M347).

However, the coding spread in this illustration (figure 3) shows that 'it was a loving experience' is almost entirely associated with 'holding the baby for a substantial time after birth'. This suggests that the BSS may account for those women who found birth to be a loving experience despite its explicit absence within the scale. In fact, on further exploration this turned out to be the case. For example, whilst the BSS doesn’t explicitly mention the transformative and spiritual nature of childbirth discussed frequently in the American 
literature (Callister, 2004; Callister \& Khalaf, 2010) , it appears to account for it through the bonding and 'natural birth' items.

Step 6. Reduction and dissemination

The data was examined in this iterative manner for further patterns, outliers, and associations. This process cycled between pattern analysis using diagrams and returning to the data in order to maintain focus on the original context.

\section{Findings and discussion}

The aim of this process was parsimony: to reduce all the data to as few as possible explanatory associations within the BSS without losing sight of the scope and range of responses. The final model was condensed from these multiple associations into 3 main themes:

\section{Being in control}

2. Being supported

3. Birth going as planned

In other words, these three BSS items explained the other associations at a general level (figure 4).

Figure 4 about here

Figure 4 suggests that the original construction of the BSS was robust. Recall that justification for the BSS items originated in the claim that satisfaction with birth was broadly correlated with quality of care provision, women's personal attributes, and stress during labour (Hollins Martin, 2008) (figure 1). It would therefore be expected that these three themes were strongly represented in the three BSS explanatory items (figure 4). Being supported is associated with quality of care provision (Fair \& Morrison, 2011). Being in control is associated with satisfaction (Berentson-Shaw, Scott, \& Jose, 2009). Stress during labour is reduced by things 'going as planned’(Hollins Martin, 2008). 
This concurrent analysis adds further weight to these connections because the literature reviewed and concurrently analysed was not part of the original literature used to devise the BSS items (Hollins-Martin \& Fleming, 2011). This demonstrates the generalisability of the BSS domains. Also, the method of concurrent analysis is essentially qualitative and therefore different in function from other methods of item analysis. The process described here can therefore be viewed as methodological triangulation (Risjord, Dunbar, \& Moloney, 2002) thus adding further rigour to the validity of the BSS. In addition, the items in figure 4 are not the same as the original themes. They are exemplars of the themes (Hollins-Martin \& Fleming, 2011)

The three main items that explained the rest were very frequently coded. They were also widely analogous with other codes. For example being communicated with was the $4^{\text {th }}$ highest frequency (see table 3 ), with a total of 151, but over half of those codes (83) were also coded at 'being supported'. Only one item is therefore necessary at a general explanatory level.

Being in control was the second highest frequency code. This was balanced to the extent that around half of the women described being out of control and half in control. Both were significant predictors of satisfaction in that being in control was coherent with items such as being well supported, calm, communicated with and receiving outstanding medical care. Likewise being out of control was associated with their opposites. This has been the subject of considerable research (Akker \& Redshaw, 2008; Baron, Cusumano, Evans, Hodne, \& Logan, 2004; Snowden et al., 2011) and adds to the evidence base that control has a significant role in satisfaction with childbirth

The third explanatory item, 'going as planned' was only the $5^{\text {th }}$ highest frequency, and this is another example of why concurrent analysis does not prioritise frequency over coherence. The next highest frequency item after control and communication/support was 'receiving much medical intervention', with 168 women mentioning this. However, the coding spread associated with this item revealed a wide range of heterogeneous responses. Receiving much medical attention was neither positive nor negative in itself. In many cases it was welcome, and in only a few was it a primary cause of distress. Where medical intervention was a cause of distress it was usually associated with the birth 'not proceeding as planned'. This is why 'going as planned' is the final major explanatory item. 
One limitation of the study is that the observations were taken at a relatively short period following birth, which is a time when postnatal women are experiencing physical and psychosocial adjustment. However, this does not render the data as inconsequential or negate its value. It would be extremely useful to undertake a longitudinal study and report observations at a variety of postnatal destinations and establish correlates with other psychometric measures, such as anxiety and depression.

The Birth Satisfaction Scale contains all the elements necessary to measure satisfaction in childbirth. Those elements that initially appeared to sit outside the framework were in fact associated with items within the scale, and could be viewed as therefore being measured by proxy.

\section{Conclusion}

The aim of this study was to examine the parsimony of the BSS with women's actual birth experience.

Results show close harmony between the BSS and the narrative data concurrently analysed. This study adds to the growing evidence for the robust and valid nature of the BSS. Implications for practice are that reports from the BSS of women's views about their birth experience will help researchers, maternity care experts and consumers construct a meaningful picture of what constitutes likes or dislikes. The BSS can be used to compare women, institutions or specific care across cultures. In addition, further statistical tests of reliability and validity will help develop a more robust BSS for measuring birth satisfaction. 


\section{References}

Akker, O. V. D., \& Redshaw, M. (2008). Beliefs and attitudes during pregnancy and post natally. Journal of Reproductive and Infant Psychology, 26(3), 155-156. doi:10.1080/02646830802224432

Baron, R. S., Cusumano, M. A., Evans, D. C., Hodne, C. J., \& Logan, H. (2004). The Effect of Desired Control and Anticipated Control on the Stress of Childbirth. Basic and Applied Social Psychology, 26(4), 249-261. Retrieved from http://www.lib.ncsu.edu/cgibin/proxy.pl?server=http://search.ebscohost.com/login.aspx?direct=true\&db=aph\&AN=15502112\&site $=$ ehost-live $\&$ scope $=$ site

Bayes, S., Fenwick, J., \& Hauck, Y. (2008). A qualitative analysis of women’s short accounts of labour and birth in a Western Australian public tertiary hospital. Journal of midwifery \& women’s health, 53(1), 53-61. doi:10.1016/j.jmwh.2007.07.021

Beck, C. T. (2006). Pentadic cartography: mapping birth trauma narratives. Qualitative Health Research, 16(4), 453-466. Retrieved from http://www.ncbi.nlm.nih.gov/pubmed/16513990

Berentson-Shaw, J., Scott, K. M., \& Jose, P. E. (2009). Do self-efficacy beliefs predict the primiparous labour and birth experience? A longitudinal study. Journal of Reproductive and Infant Psychology, 27(4), 357-373. doi:10.1080/02646830903190888

Callister, L. C. (2004). Making meaning: women’s birth narratives. Journal of obstetric gynecologic and neonatal nursing JOGNN NAACOG, 33(4), 508-518. Retrieved from http://doi.wiley.com/10.1177/0884217504266898

Callister, L. C., \& Khalaf, I. (2010). Spirituality in childbearing women. The Journal of perinatal education, 19(2), 16-24. doi:10.1624/105812410X495514

Charmaz, K. (2009). Developing Grounded Theory: The Second generation. In J. Morse, P. Noerager Stern, J. Corbin, B. Bowers, K. Charmaz, \& E. Clarke (Eds.), (pp. 127-149). Walnut Creek, CA: Left Coast Press. 
Cutcliffe, J. R., \& Harder, H. G. (2009). The perpetual search for parsimony: enhancing the epistemological and practical utility of qualitative research findings. International journal of nursing studies, 46(10), 1401-10. doi:10.1016/j.ijnurstu.2009.05.005

Fair, C. D., \& Morrison, T. E. (2011). The relationship between prenatal control, expectations, experienced control, and birth satisfaction among primiparous women. Midwifery, 1-6. Elsevier. doi:10.1016/j.midw.2010.10.013

Flemming, K. (2010). Synthesis of quantitative and qualitative research: an example using Critical Interpretive Synthesis. Journal of advanced nursing, 66(1), 201-17. doi:10.1111/j.13652648.2009.05173.x

Hollins Martin, C. J. (2008). Birth planning for midwives and mothers, 583-588. MA Healthcare Ltd. Retrieved from http://researchonline.gcu.ac.uk/health/196/

Hollins-Martin, C., \& Fleming, V. (2011). The birth satisfaction scale. International Journal of Health Care Quality Assurance, 24(2), 124-135. doi:10.1108/09526861111105086

Redshaw, M. (2008). Women as consumers of maternity care: measuring “satisfaction” or “dissatisfaction”? Birth Berkeley Calif, 35(1), 73-76. Retrieved from http://www.ncbi.nlm.nih.gov/pubmed/18307491

Risjord, M. W., Dunbar, S. B., \& Moloney, M. F. (2002). A new foundation for methodological triangulation. Journal of nursing scholarship an official publication of Sigma Theta Tau International Honor Society of Nursing Sigma Theta Tau, 34(3), 269-275. Retrieved from http://www.ncbi.nlm.nih.gov/pubmed/12237990

Shattell, M. M., McAllister, S., Hogan, B., \& Thomas, S. P. (2006). "She took the time to make sure she understood": mental health patients' experiences of being understood. Archives of psychiatric nursing, 20(5), 234-41. doi:10.1016/j.apnu.2006.02.002

Snowden, A., \& Atkinson, J. (2012). Concurrent analysis: a pragmatic justification. Nursing Philosophy, 13, 126-141. 
Snowden, A., \& Martin, C. (2010a). Concurrent analysis : towards generalisable qualitative research. Journal of Clinical Nursing. doi:10.1111/j.1365-2702.2010.03513.x

Snowden, A., \& Martin, C. R. (2010b). Mental health nurse prescribing: a difficult pill to swallow? Journal of psychiatric and mental health nursing, 17(6), 543-53. doi:10.1111/j.1365-2850.2010.01561.x

Snowden, A., Martin, C. R., Jomeen, J., \& Hollins Martin, C. J. (2011). Concurrent analysis of choice and control in childbirth. BMC pregnancy and childbirth, 11(1), 40. doi:10.1186/1471-2393-11-40

Thagard, P. (2007). Coherence, Truth, and the Development of Scientific Knowledge*. Philosophy of Science, 74(1), 28-47. doi:10.1086/520941

Thorne, S. (2011). Toward methodological emancipation in applied health research. Qualitative health research, 21(4), 443-53. doi:10.1177/1049732310392595 